\title{
Collagen-Based Fuel Cell and Its Proton Transfer
}

\author{
Yasumitsu Matsuo, Hiroki Ikeda, Takashi Kawabata, Junko Hatori, Hiroshi Oyama \\ Department of Life Science, Faculty of Science \& Engineering, Setsunan University, Neyagawa, Japan \\ Email: ymatsuo@lif.setsunan.ac.jp
}

How to cite this paper: Matsuo, Y., Ikeda, H., Kawabata, T., Hatori, J. and Oyama, $\mathrm{H}$. (2017) Collagen-Based Fuel Cell and Its Proton Transfer. Materials Sciences and Applications, 8, 747-756. https://doi.org/10.4236/msa.2017.811054

Received: September 3, 2017

Accepted: October 7, 2017

Published: October 10, 2017

Copyright $\odot 2017$ by authors and Scientific Research Publishing Inc. This work is licensed under the Creative Commons Attribution International License (CC BY 4.0).

http://creativecommons.org/licenses/by/4.0/

(c) (i) Open Access

\begin{abstract}
We have fabricated the fuel cell based on the tissue derived biomaterial "collagen" and investigated its proton transfer. It was found that "collagen" becomes the electrolyte of fuel cell in the humidified condition. The power density of the fuel cell becomes typically $8.6 \mathrm{~W} / \mathrm{m}^{2}$ in the $80 \%$ humidity. Further, these results indicate that collagen exhibits proton conductivity in the humidified condition. Both of proton conductivity and dielectric constant increase by the increase of humidity. From the analyses of the frequency dependence of AC conductivity, it was found that proton conductivity and the dielectric dispersion observed in the humidified condition are caused by the formation of the water bridge, which is bonded with the collagen peptide chain. Considering that hydration induces the formation of the water bridge and that increases proton conductivity and dielectric constant, it is deduced that proton transfer in the fuel cell based on collagen is caused by the breaking and rearrangement of hydrogen bond in the water bridge.
\end{abstract}

\section{Keywords}

Fuel Cell, Proton Conductor, Electrolyte, Biomaterials

\section{Introduction}

It is well-known that tissue-derived biomaterials are abundant in nature and are widely used in biomedical field. Especially, it is known that these materials are used to the formation of artificial blood vessel, artificial bone filling material and so on. Recently, even in the field of electrical conductor, the application of tissue-derived biomaterials to devices begins to investigate, because the tissue-derived biomaterials have the features of charge transfer such as proton pump and ion channel, and further have a potential to achieve a low-carbon society. For example, the electrical properties of deoxyribo-nucleic acid (DNA) 
have been investigated by many researchers, and there are many reports [1]-[7]. For example, in the field of electrical devices, the investigations of DNA molecular wire and DNA based materials for switching devices have been carried out [8] [9]. It is also known that the DNA film exhibits proton conductivity in the humidified condition and can be used as the electrolyte of fuel cell [10] [11]. In this way, tissue-derived biomaterials are useful for the application to electrical devices and become excellent proton-transfer materials. These facts mean that tissue-derived biomaterials are important material in the field of "Protonics". Recently, we have fabricated the fuel cell based on the electrolyte of submucosa film, which is the part of intestine [12]. It was found that the current density $i$ dependence of cell voltage $V$ ( $i-V$ curve) of fuel cell based on submucosa in the humidified condition shows a typical $i$ - $V$ characteristics of fuel cell [12]. The maximum power density in its fuel cell reaches to $1.4 \mathrm{~W} / \mathrm{m}^{2}$ and is stable for 30 days at least. This result indicates that submucosa becomes the electrolyte of fuel cell and that becomes proton conductor in the humidified condition. It is well-known that submucosa film mainly consists of collagen which is one of biopolymers. In the present work, we have shown the possibility to the fuel cell based on the biopolymer "collagen" electrolyte and investigated the important factor for the appearance of proton conductivity. It is expected that these results will be helpful to the development to the electrical devices and energy devices of biopolymer, such as the power supply of artificial heart.

\section{Experimental}

\subsection{Sample Preparation}

The films of collagen were prepared from the decalcified scales of Tilapia fish (Nitta Gelatin Inc). Figure 1 shows the photograph of the collagen film. The thickness of its film is approximately $0.25 \mathrm{~mm}$. In the fabrication of fuel cell, the collagen film was used as an electrolyte. In the impedance measurement, the electrical conductivity perpendicular to the surface of collagen film was measured.

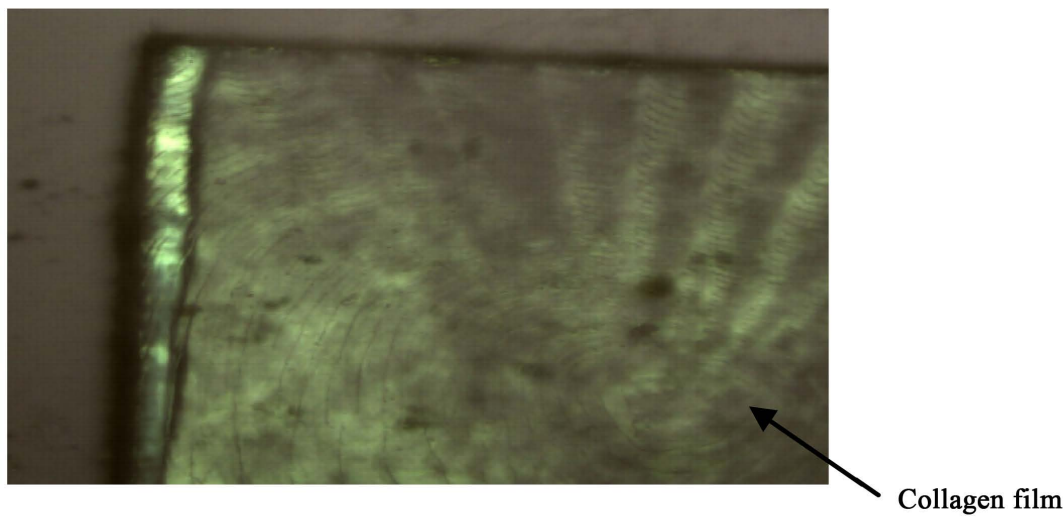

Figure 1. Photograph of collagen film. 


\subsection{Fabrication of Fuel Cell Based on Collagen Electrolyte}

Figure 2 shows the shape of fuel cell based on the collagen electrolyte. The collagen electrolyte was sandwiched by the Pt-C catalyst electrodes (anode and cathode). The current was collected from the current collector plate. The hydrogen gas was introduced from the center of the fuel cell and was injected in the left sides of fuel cell. The oxygen gas was introduced in the right side of fuel cell. The relative humidity and fuel gas flow were controlled by the humidified gas-flow control system of Auto PEM (Toyo Corporation).

\subsection{Impedance Analysis}

For the impedance measurement, the admittance mode which consists of the parallel circuit of capacitance and conductance was used as the equivalent circuit of collagen film. In this case, the electrical conductivity $\sigma_{\mathrm{AC}}$ is calculated from the simple equation of $\sigma_{\mathrm{AC}}=\omega \varepsilon_{0} \varepsilon^{\prime \prime}$, where $\omega$ is angular frequency, $\varepsilon^{\prime \prime}$ and $\varepsilon_{0}$ are the imaginary part of complex dielectric constant and the dielectric constant in vacuum, respectively. In the case that $\sigma_{\mathrm{AC}}$ is proportional to $\omega$, the equivalent circuit corresponds to the simplest equivalent circuit which consists of the parallel circuit of capacitor and resistor.

On the other hand, when the measured AC conductivity is not proportional to $\omega$ and shows the complex frequency dependence, we cannot use the simple equation $\sigma_{\mathrm{AC}}=\omega \mathcal{E}_{0} \mathcal{E}^{\prime \prime}$, because the AC electrical conductivity includes the components of dielectric dispersion. The AC conductivity including dielectric dispersion is described by the following equation:

$$
\begin{aligned}
\sigma_{\mathrm{AC}} & =\sigma_{\mathrm{DC}}-\operatorname{Im}\left[\omega \varepsilon_{0} \varepsilon_{\infty}+\frac{\omega \varepsilon_{0}\left(\varepsilon_{s}-\varepsilon_{\infty}\right)}{1+(j \omega \tau)^{\beta}}\right] \\
& =\sigma_{\mathrm{DC}}+\frac{\omega \varepsilon_{0}\left(\varepsilon_{s}-\varepsilon_{\infty}\right)(\omega \tau)^{\beta} \sin \left(\frac{\pi}{2} \beta\right)}{\left(1+(\omega \tau)^{\beta} \cos \left(\frac{\pi}{2} \beta\right)\right)^{2}+\left((\omega \tau)^{\beta} \sin \left(\frac{\pi}{2} \beta\right)\right)^{2}}
\end{aligned}
$$

where $\sigma_{\mathrm{DC}}$ is DC electrical conductivity, $\mathcal{E}_{\mathrm{s}}$ and $\mathcal{E}_{\infty}$ are the static and unrelaxed dielectric constants, respectively, $\tau$ and $\beta$ are the relaxation time for dielectric

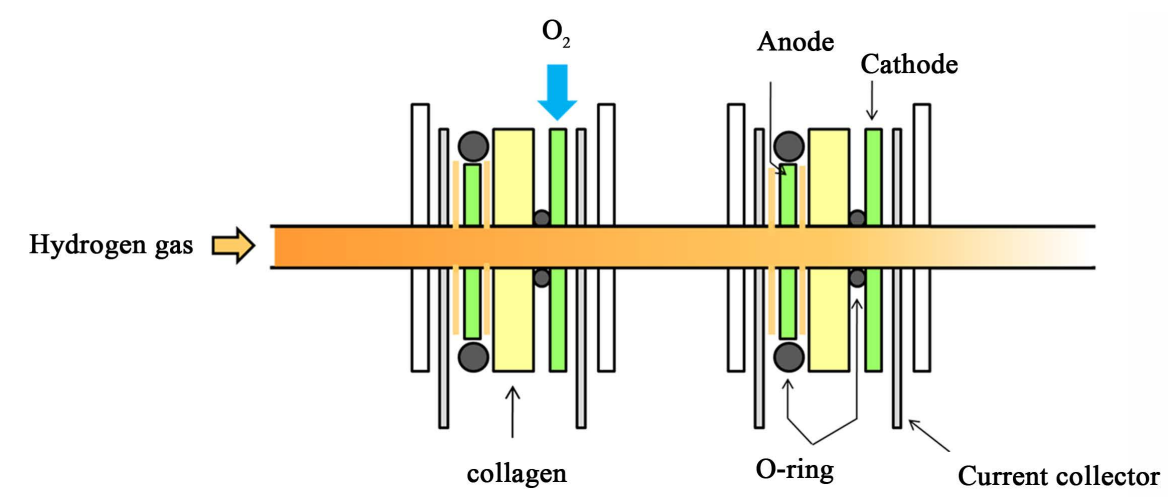

Figure 2. Collagen based fuel cell (two single cells). 
dispersion and the degree of mono-dispersion, respectively. The second term of Equation (1) shows the component of dielectric dispersion and the step-like anomaly appears in the frequency dependence of AC electrical conductivity. In other words, we can obtain the motion of molecules causing the dielectric dispersion from the frequency dependence of AC conductivity using Equation (1). The AC electrical conductivity measurement was carried out using precision LCR meter (E4980A, Agilent Technologies Inc.).

\section{Results and Discussion}

Figure 3 shows the current density $i$ versus cell voltage $V_{\text {cell }}$ curve ( $i-V_{\text {cell }}$ curve) of fuel cell based on the collagen electrolyte in the $80 \%$ relative humidity. It is evident that $i-V_{\text {cell }}$ curve in Figure 3 shows the typical $i-V$ characteristic feature of fuel cell with the slight over-potential effect at around the very low-current region. Considering that this $i-V_{\text {cell }}$ curve is observed by introducing the hydrogen gas, this result indicates that the collagen becomes the electrolyte of fuel cell. The maximum power density in collagen is $8.6 \mathrm{~W} / \mathrm{m}^{2}$. This value is about 6 times larger than the maximum power density $1.4 \mathrm{~W} / \mathrm{m}^{2}$ of fuel cell based on submucosa electrolyte which consists of collagen and cellulose [12]. This result implies that the power generation is caused by the collagen which is mainly included in both the scale of fish and submucosa.

Figure 4 shows the operation of digital clock by using the collagen-based fuel cell in the $100 \%$ relative humidity. As shown in Figure 4, it is evident that digital clock actually operates by three collagen-based fuel cells. In this way, the biomaterials have a potential as the electrolyte which is the most important part in

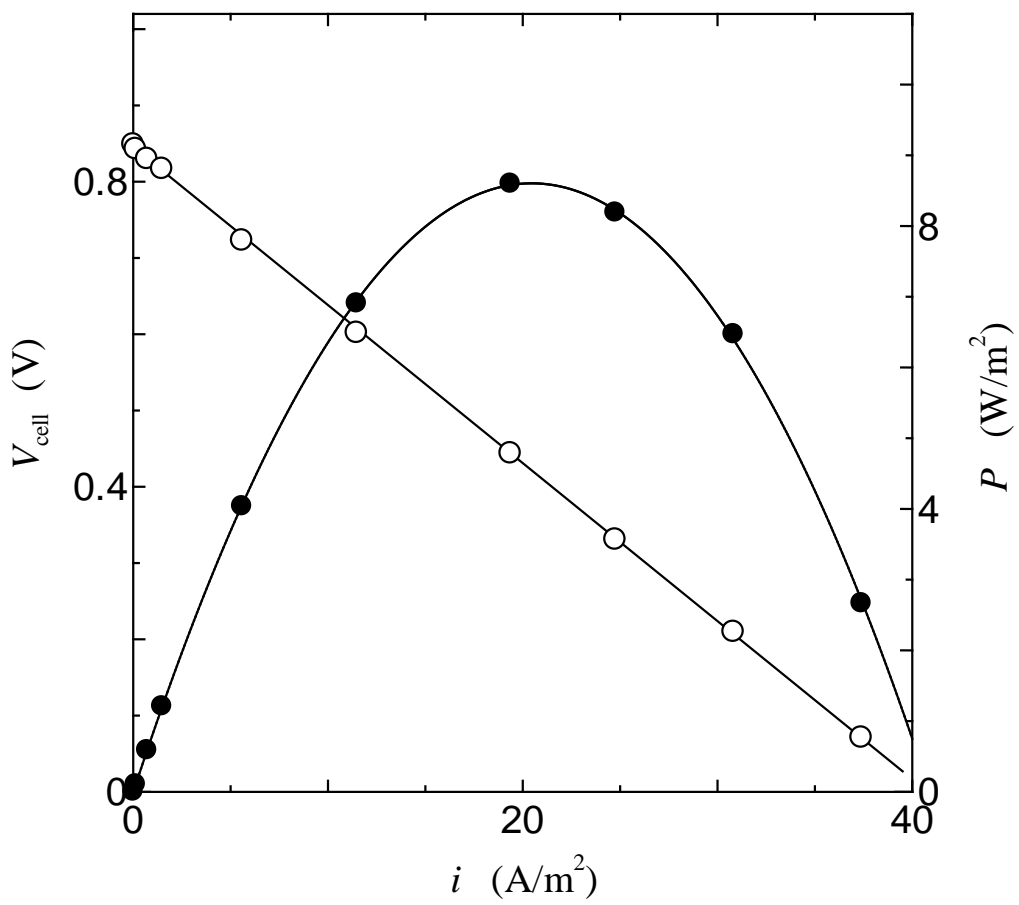

Figure 3. Current density versus cell voltage in collagen-based fuel cell. 


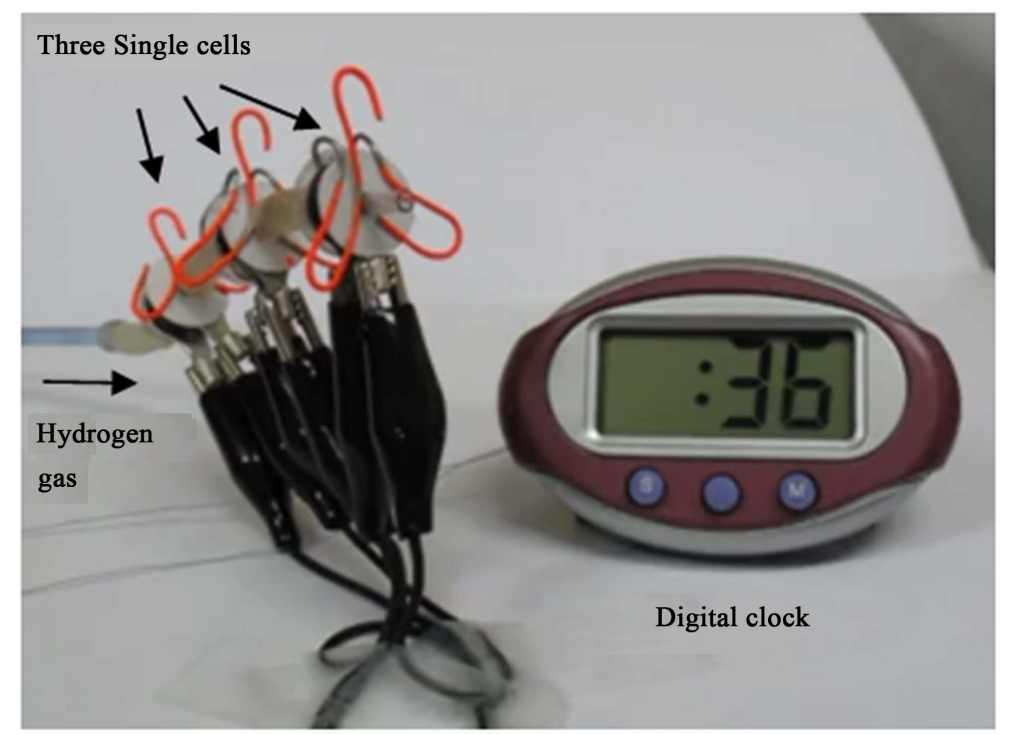

Figure 4. State of the operation of digital clock using the collagen-based fuel cell.

fuel cell. In addition, these results in Figure 3 and Figure 4 indicate that the collagen exhibits proton conductivity in the humidified condition.

In order to investigate the key factor for the appearance of proton conductivity, we have carried out the impedance measurements. Figure 5 shows the frequency dependence of AC electrical conductivity $\sigma_{\mathrm{AC}}$ in collagen with a parameter of relative humidity. The value of $\sigma_{\mathrm{AC}}$ increases with the increase in relative humidity. Moreover, it is noted that electrical conductivity in the higher humidity condition shows not the monotonous increase with the increase in frequency but the small-step anomaly with the increase in frequency. This result shows that electrical conductivity $\sigma_{\mathrm{AC}}$ includes not only the component of DC electrical conductivity but also the component of dielectric dispersion due to the dielectric polarization which is caused by the humidification. Further, the frequency, where small-step anomaly is observed, decreases with the increase in humidity. These results indicate that the dielectric loss with the relaxation frequency, which corresponds to the frequency of the small-step anomaly, appears by the increase in humidity.

As described above, the AC conductivity including the dielectric dispersion is described by Equation (1). The AC conductivity $\sigma_{\mathrm{AC}}$ calculated from Equation (1) is shown in Figure 5 as solid lines. The values of $\sigma_{\mathrm{DC}}, \varepsilon_{\mathrm{s}}-\varepsilon_{\infty}, \tau$ and $\beta$ used in the calculation of solid line in Figure 5 are listed in Table 1.

As shown in Figure 5, we can clearly see that the calculated curves are in good agreement with experimental results. These results mean that the measured $\sigma_{\mathrm{AC}}$ is described by Equation (1). Figure 6(a) shows the humidity dependence of DC proton conductivity $\sigma_{\mathrm{DC}}$ obtained from Figure 5 and Equation (1). As shown in Figure $6(\mathrm{a}), \sigma_{\mathrm{DC}}$ increases by increasing relative humidity, shows the change of slope at around $80 \%$ relative humidity and thereafter increases. This result indicates that proton conducting path increases with the increase in the relative 
Table 1. Values of DC conductivity and dielectric dispersion parameters.

\begin{tabular}{ccccc}
\hline Relative humidity (\%) & $\sigma_{D C}$ & $\mathcal{E}_{s}-\varepsilon_{\infty}$ & $\tau$ & $\beta$ \\
\hline 100 & $4.0 \times 10^{-3}$ & $2.0 \times 10^{3}$ & $1.0 \times 10^{-6}$ & 0.40 \\
81 & $5.0 \times 10^{-5}$ & $9.1 \times 10^{2}$ & $1.9 \times 10^{-5}$ & 0.60 \\
53 & $1.0 \times 10^{-5}$ & $8.7 \times 10^{2}$ & $8.1 \times 10^{-5}$ & 0.63
\end{tabular}

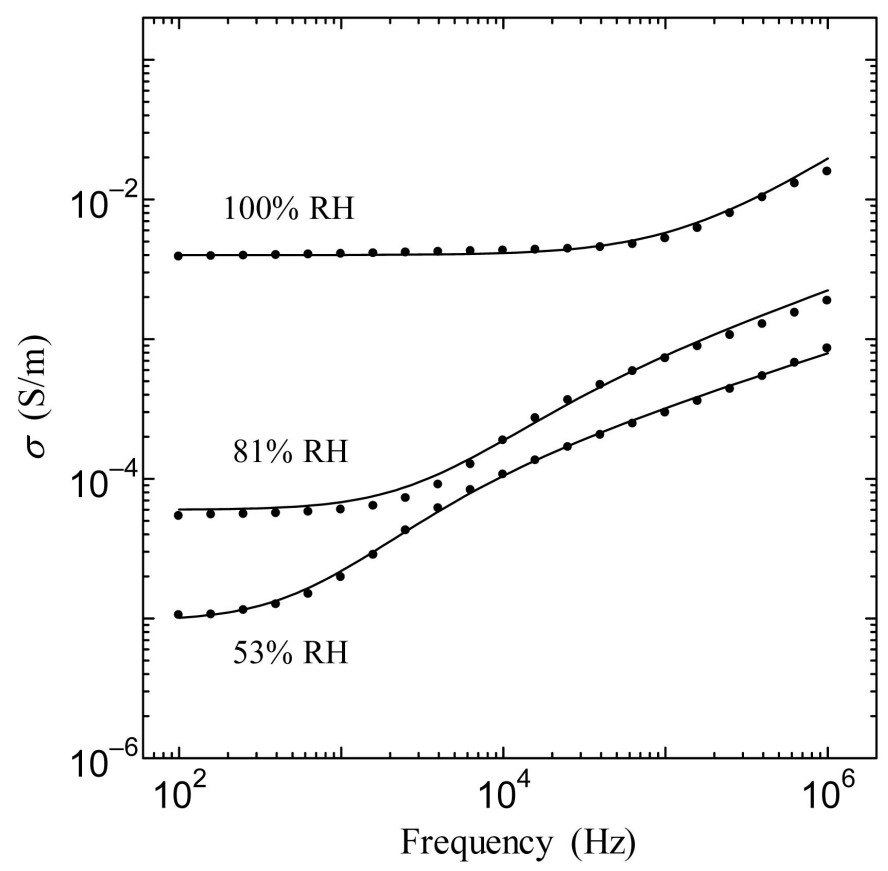

Figure 5. Frequency dependence of $\sigma_{\mathrm{AC}}$ in collagen.

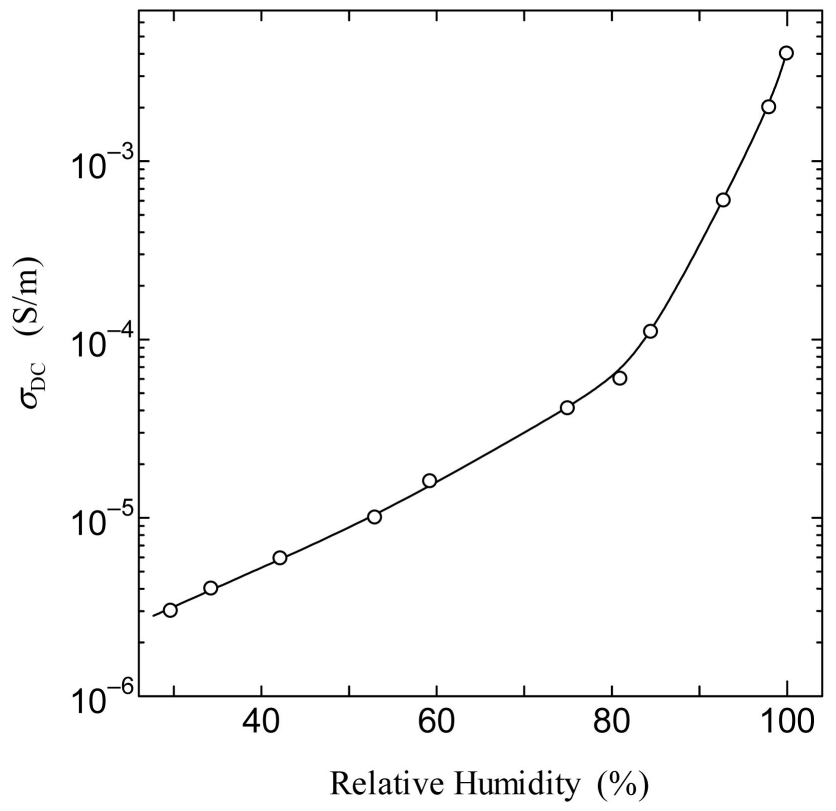

(a)

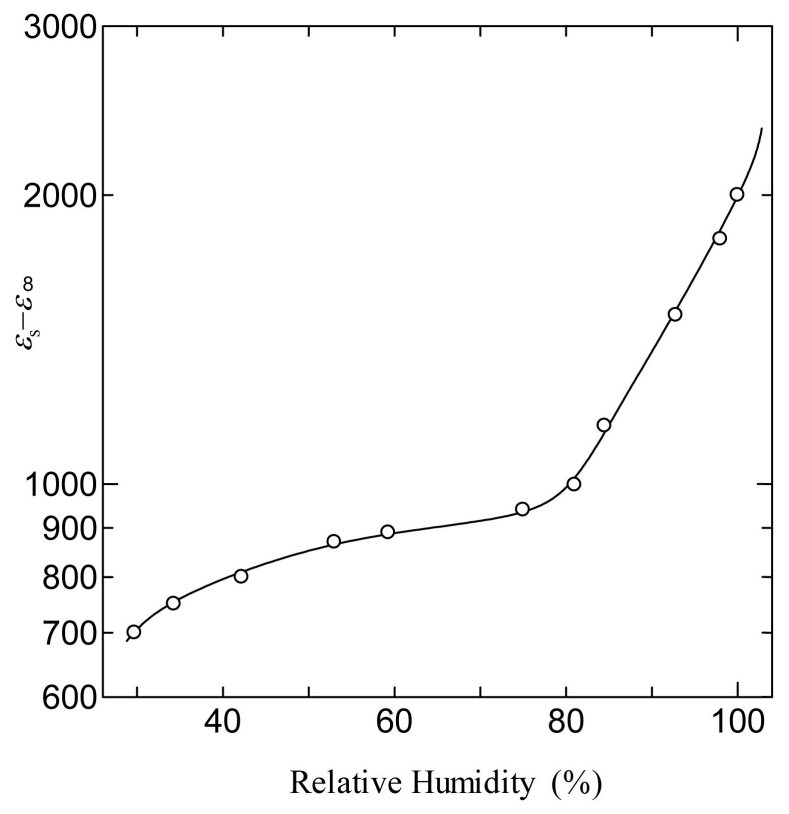

(b)

Figure 6. Relative humidity dependences of $\sigma_{\mathrm{DC}}(\mathrm{a})$ and $\varepsilon_{\mathrm{s}}-\varepsilon_{\infty}(\mathrm{b})$. 
humidity. Figure 6(b) shows the humidity dependence of static dielectric constant $\mathcal{E}_{\mathrm{s}}-\varepsilon_{\infty}$. The static dielectric constant $\varepsilon_{\mathrm{s}}-\varepsilon_{\infty}$ also increases by increasing relative humidity and shows the change of slope at the $80 \%$ relative humidity where the slope of $\sigma_{\mathrm{DC}}$ changes. These consistencies of the behaviors of $\sigma_{\mathrm{DC}}$ and $\varepsilon_{\mathrm{s}}-\varepsilon_{\infty}$ indicate that the water molecule injected into collagen causes not only the change in proton conductivity but also the change in dielectric constant. Therefore, these results indicate that the water molecules lead to not only the appearance of proton conducting path but also of dielectric polarization. In addition, we can see clearly that in the present case the long dielectric-relaxation times $\tau$ $\left(10^{-6}-10^{-5} \mathrm{sec}\right)$ are observed. It is known that the relaxation time $\tau$ of $10^{-6}$ to $10^{-5} \mathrm{sec}$ is observed when the cooperated motion seen in " $\alpha$ relaxation" exists between protein and water molecule [13] [14] [15] [16] [17]. These results indicate that the long relaxation time observed in the present case is caused by the relaxation of the cooperated motion between collagen peptide and water molecule generated by humidifying. It is also known that, in the humidified condition, collagen molecule forms the water bridges [18]. As an example, we show the schematic structure for the collagen peptide chain with the water bridges in Figure 7. As shown in Figure 7, in the humidified condition, collagen has the water bridges of inter- or intra-collagen peptide chains. Considering that the water bridges strongly affect the proton conductivity and dielectric properties and that cause the $\alpha$ relaxation, it is deduced that these water bridges in collagen lead to the increases of $\sigma_{\mathrm{DC}}$ and $\varepsilon_{\mathrm{s}}-\varepsilon_{\infty}$ in the humidified condition. Figure 8 shows the example of proton transfer in the water bridge between the collagen peptide chains. The water bridge is constructed by the bonding between the water molecule and hydrophile molecules (hydroxylproline or glycine carbonyl group) in the collagen peptide. It is well known that, in the hydrogen bond, hydronium ions will be generated, because hydroxyproline and glycine (amino

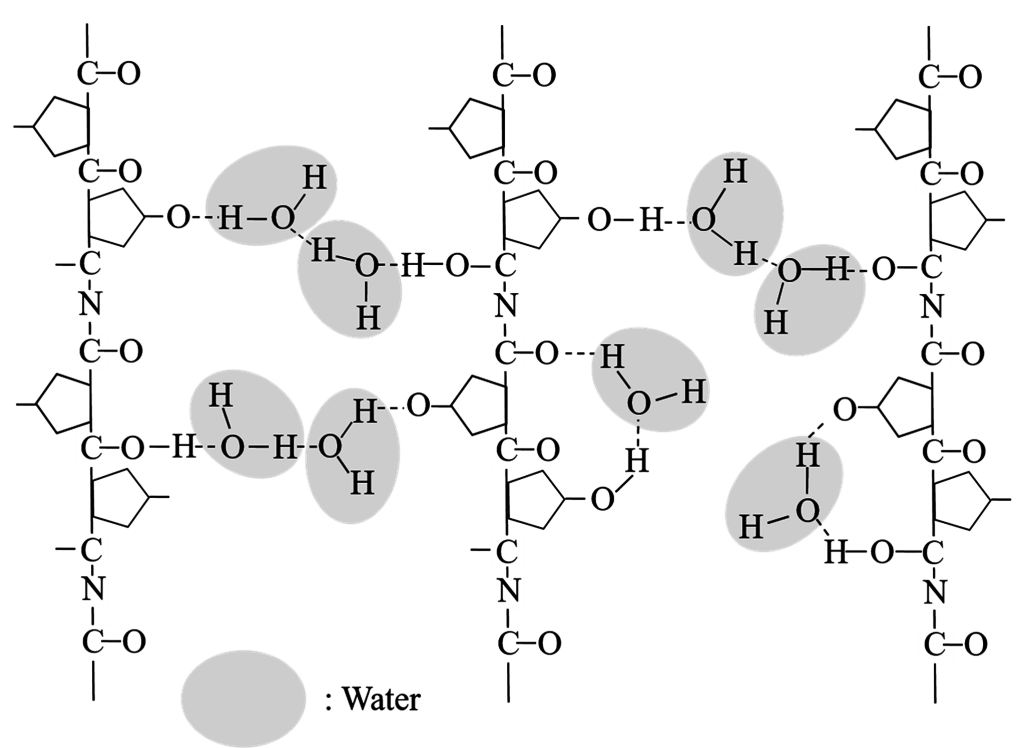

Figure 7. Schematic structure for collagen peptide chain with the water bridges. 
(a)

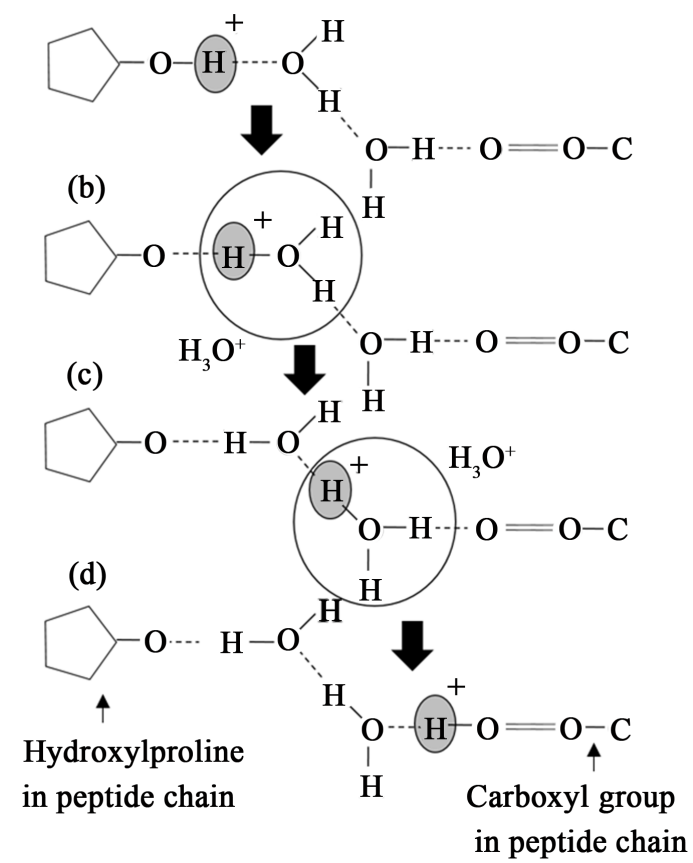

Figure 8. Example of proton transfer in the water bridge between collagen peptide chains.

acid) are easily able to give and take protons of water molecule in the water bridge. The generated hydronium ions moves with the proton transfer in the water bridge, as shown in Figure 8(b) and Figure 8(c) and consequently proton moves from one peptide chain to another peptide chain. In this way, water bridges cause the proton transfer.

Considering these results, it is deduced that mobile protons appear by the breaking and rearrangement of hydrogen bond in the water bridges and then proton conductivity and large dielectric polarization are realized. In the present work, it is also found that the slope of the humidity dependences of $\sigma_{\mathrm{DC}}$ and $\mathcal{E}_{\mathrm{s}}-$ $\mathcal{E}_{\infty}$ changes at the $80 \%$ relative humidity. This anomaly may be caused by the hydration-induced phase transition. In order to clarify the anomalous behavior of $\sigma_{\mathrm{DC}}$ and $\mathcal{E}_{\mathrm{s}}-\varepsilon_{\infty}$ at the $80 \%$ relative humidity, we are now planning the investigation of the structure change at the $80 \%$ relative humidity by neutron diffraction measurement. These results will appear future issues.

\section{Conclusion}

In the present paper, it was found that collagen becomes the electrolyte of fuel cell and that the collagen based fuel cell shows the power density of typically 8.6 $\mathrm{W} / \mathrm{m}^{2}$ in the $80 \%$ humidity. These results also indicate that collagen exhibits proton conductivity in the humidified condition. From the humidity dependences of proton conductivity and dielectric constant, proton conductivity but also dielectric increase by the increase in humidity. From the analyses of the frequency dependence of AC conductivity, it was found that the dielectric dis- 
persion observed in the humidified condition is caused by the formation of the water bridge, which is bonded with the collagen peptide chain. Considering that hydration induces the formation of the water bridge and that increases proton conductivity and dielectric constant, it is deduced that proton transfer in collagen is caused by the formation of the water bridge and that proton transfer is realized by the breaking and rearrangement of proton in water bridge.

\section{Acknowledgements}

This study was supported by the Adaptable and Seamless Technology transfer Program through target-driven R\&D from Japan Science and Technology Agency.

\section{References}

[1] Fink, H.-W. and Schenenberger, C. (1999) Electrical Conduction through DNA Molecules. Nature, 398, 407-410. https://doi.org/10.1038/18855

[2] Porath, D., Bezryadin, A., Simon de Vries and Dekker, C. (2000) Direct Measurement of Electrical Transport through DNA Molecules. Nature, 403, 635-638.

https://doi.org/10.1038/35001029

[3] Wilson, E. (2001) Ion Channels in Membranes Aid Oligonucleotide Analysis. Chemical \& Engineering News, 79, 20. https://doi.org/10.1021/cen-v079n013.p020

[4] Kasumov, A. Y., Kociak, M., Gueron, S., Reulet, B., Volkov, V.T., Klinov, D.V. and Bouchiate H. (2001) Proximity-Induced Superconductivity in DNA. Science, 291, 280-282. https://doi.org/10.1126/science.291.5502.280

[5] Kutnjak, Z., Filipic, C., Podgornik, R., Norgenskiold, L. and Korolev, N. (2003) Electrical Conduction in Native Deoxyribonucleic Acid: Hole Hopping Transfer Mechanism? Physical Review Letters, 90, 98101-98104. https://doi.org/10.1103/PhysRevLett.90.098101

[6] Seeman, N.C. (2003) DNA in a Material World. Nature, 421, 427-431. https://doi.org/10.1038/nature01406

[7] Matsuo, Y., Sugita, K. and Ikehata, S. (2005) Doping Effect for Ionic Conductivity in DNA Film. Synthetic Metals, 154, 13-16. https://doi.org/10.1016/j.synthmet.2005.07.004

[8] Campolongo, M.J., Kahn, J.S., Cheng, W., Yang, D., Gupton-Campolongo, T. and Luo, D. (2011) Adaptive DNA-Based Materials for Switching, Sensing, and Logic Devices. Journal of Materials Chemistry, 21, 6113-6121. https://doi.org/10.1039/c0jm03854g

[9] Wohlgamuth, C.H., McWilliams, M.A. and Slinker, J.D. (2013) DNA as a Molecular Wire: Distance and Sequence Dependence. Analytical Chemistry, 85, 8634-8640. https://doi.org/10.1021/ac401229q

[10] Matsuo, Y., Kumasaka, G., Saito, K. and Ikehata, S. (2004) Fabrication of Solid-State Fuel Cell Based on DNA Film. Solid State Communications, 133, 61-64. https://doi.org/10.1016/j.ssc.2004.09.055

[11] Matsuo, Y., Hatori, J., Yoshida, Y. and Ikehata, S. (2010) Humidity Dependence of Proton Conductivity in DNA Film Studied by NMR and AC Conductivity. Journal of the Physical Society of Japan, Suppl. A79, 12-14. https://doi.org/10.1143/JPSJS.79SA.12 
[12] Matsuo, Y. and Hatori, J. (2011) Fuel Cell Based on Natural Sausage Casing. Journal of Biobased Materials and Bioenergy, 5, 562-564. https://doi.org/10.1166/jbmb.2011.1173

[13] Davis, R.D., Bur, A.J., McBrearty, M., Lee, Y.-H., Gilman, J.W. and Start, P.R. (2004) Dielectric Spectroscopy during Extrusion Processing of Polymer Nanocomposites: A High Throughput Processing/Characterization Method to Measure Layered Silicate Content and Exfoliation. Polymer, 45, 6487-6493. https://doi.org/10.1016/j.polymer.2004.07.047

[14] Sengwa R.J. and Choudhary, S. (2010) Investigation of Correlation between Dielectric Parameters and Nanostructures in Aqueous Solution Grown Poly(Vinylalcohol)Montmorillonite Clay Nanocomposites by Dielectric Relaxation Spectroscopy. EXpress Polymer Letters, 4, 559-569. https://doi.org/10.3144/expresspolymlett.2010.70

[15] Shinyashiki, N., Imoto, D. and Yagihara, S. (2007) Broadband Dielectric Study of Dynamics of Polymer and Solvent in Poly(Vinyl Pyrrolidone)/Normal Alcohol Mixtures. The Journal of Physical Chemistry B, 111, 2181-2187. https://doi.org/10.1021/jp065414e

[16] Shinyashiki, N., Sudo, S., Yagihara, S., Spanoudaki, A., Kyritsis, A. and Pissis, P. (2007) Relaxation Processes of Water in the Liquid to Glassy States of Water Mixtures Studied by Broadband Dielectric Spectroscopy. Journal of Physics: Condensed Matter, 19, 205113-205125. https://doi.org/10.1088/0953-8984/19/20/205113

[17] Cerveny, A., Alegría, A. and Colmenero, J. (2008) Universal Features of Water Dynamics in Solutions of Hydrophilic Polymers, Biopolymers, and Small Glass-Forming Materials. Physical Review E, 77, Article ID: 031803. https://doi.org/10.1103/PhysRevE.77.031803

[18] Bella, J., Brodsky, B. and Berman, H.M. (1995) Hydration Structure of a Collagen Peptide. Structure, 3, 893-906. https://doi.org/10.1016/S0969-2126(01)00224-6 